DOI: 10.15593/RZhBiomeh/2016.2.04

УДК $539.3+617.3+616-001$

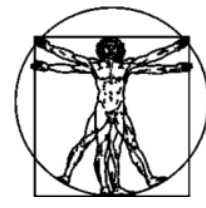

Росеийекий Журнал Биомеханики

www.biomech.ru

\title{
БИОМЕХАНИКА РАЗГРУЗКИ НИЖНИХ КОНЕЧНОСТЕЙ ПРИ ОРТЕЗИРОВАНИИ
}

\author{
И.Н. Дашевский ${ }^{1}$, С.Е. Никитин ${ }^{2}$ \\ 1 Лаборатория механики прочности и разрушения материалов и конструкций Института проблем механики \\ им. А.Ю. Ишлинского РАН, Россия, 119526, Москва, проспект Вернадского, 101-1, e-mail: dash@ipmnet.ru \\ ${ }^{2}$ Медицинское отделение Федерального государственного унитарного предприятия «Центральный \\ научно-исследовательский институт травматологии и ортопедии им. Н.Н. Приорова» Минздрава РФ, \\ Россия, 127299, Москва, ул. Приорова, 10, e-mail: runiks@list.ru
}

\begin{abstract}
Аннотация. Изучается биомеханика одного типа современных ортезов нижних конечностей - ортопедических аппаратов, дающих возможность гибкой подстройки под конкретного пациента в течение всего периода лечения, что позволяет обеспечить лучшую фиксацию и разгрузку проблемной зоны, уменьшить сроки и улучшить качество лечения. Поскольку перегрузка поврежденного участка чревата осложениями, а его недогрузка приводит к ослаблению и атросрии костномышечного аппарата и удлинению сроков реабилитации, важное значение имеет исследование разгрузки конечности при ортезировании, что и составляет главную цель работы. Развивается комбинированный расчетно-экспериментальный подход к изучению биомеханики системы нога-тутор. В эксперименте in vivo продемонстрирован эффект возникновения разгрузки нижней конечности при ее ортезировании для ортезов двух типов. Вводится количественная характеристика разгрузки - коэффициент разгрузки, рассчитываемый как отношение максимальной нагрузки на неортезированную конечность к максимальной нагрузке на конечность в ортезе. Описаны прямые экспериментальные измерения коэффициента разгрузки in vivo c помощью программно-аппаратного комплекса «ДиаСлед» для двух видов ортезов и при разной степени бокового обжатия (оцениваемого на качественном уровне). Для коэффициента разгрузки получены значения от 1 до примерно 2,3 в зависимости от конкретных условий. На основе сравнительных оценок жесткости отдельных элементов системы нога-ортез предложена минимальная базовая биомеханическая модель разгрузки конечности в ортезе и получено соответствующее ей выражение для коэффициента разгрузки. Модель дает для коэффициента разгрузки оценку порядка 1,6...2,1 при отсутствии боковой компрессии (в зависимости от условий контакта конечности с ортезом) и постоянство либо линейное падение нагрузки на конечность с ростом обжатия.
\end{abstract}

Ключевые слова: биомеханика конечностей, ортезы, туторы, нижние конечности, экспериментальные исследования, разгрузка, ДиаСлед.

\section{ВВЕДЕНИЕ}

Появление в последние 30 лет новых материалов обусловило постепенное вытеснение простых средств типа гипсовых повязок и шин ортопедическими конструкциями нового типа - высокотехнологичными ортезами. Последние дают возможность подтяжки и гибкой подстройки под конкретного пациента в течение всего периода лечения, что позволяет обеспечить лучшую фиксацию и разгрузку проблемной 
зоны, уменьшить сроки и улучшить качество лечения. Ортез (греч. orthos - прямой, правильный) - специальное техническое устройство (серийного или индивидуального изготовления), применяемое для фиксации, разгрузки, коррекции и активизации функций поврежденной конечности. В широком смысле термин ортез включает в себя весь спектр ортопедических приспособлений: от наколенников и стелек до корсетов, туторов, аппаратов и т.д. [20, 24].

Одной из основных функций ортезов является разгрузка поврежденного участка кости $[10,15,17]$. Полная нагрузка конечности до наступления консолидации может привести к осложнениям и повторным повреждениям проблемного участка $[1,6]$, в то же время чрезмерная разгрузка (например с помощью гипсовых повязок) имеет следствием заметное ослабление и атрофию костно-мышечного аппарата и удлинение сроков реабилитации $[11,16]$. Поэтому, очевидно, существует некоторый оптимальный уровень (точнее программа) разгрузки, при котором, с одной стороны, снижена опасность повторных повреждений, а с другой - обеспечена возможность проведения динамической коррекции поврежденного сегмента в функционально-правильном положении, что способствует восстановлению локомоторной функции конечности и ранней реабилитации в максимально комфортных условиях при различных локализации и характере повреждения $[13,14]$. Это определяет важность и актуальность проблемы изучения механизмов разгрузки и возможностей управления ею [18].

Несмотря на растущее использование ортезов в клинике, спорте и быту и наличие ряда медицинских руководств и атласов по ортезам $[34,38]$, направление, связанное с механико-математическим моделированием системы нога-ортез, находится лишь в начале своего развития. Публикации, касающиеся моделирования ортезирования собственно конечностей (биомеханика стопы и стелек фактически составляет отдельное самостоятельное направление исследований) методами механики деформируемого твердого тела, пока единичны, да и те касаются лишь ортеза как такового и совершенно игнорируют саму конечность и ее механические свойства $[30,31,37]$. На данном этапе развития этого направления предпочтительным представляется комбинированный оценочно-экспериментальный подход, включающий в себя в том или ином порядке (и при необходимости в несколько циклов): a) выяснение основных качественных особенностей системы и получение грубых оценок на возможно более простых биомеханических моделях; б) прямую экспериментальную проверку полученных выводов и результатов и прямое экспериментальное измерение интересующих величин. Целью данного исследования как раз и является экспериментальное измерение и биомеханическое моделирование разгрузки нижних конечностей при ортезировании в указанном стиле. В настоящей работе излагаются начальные результаты изучения степени разгрузки пораженной области при использовании двух видов ортезов нижних конечностей и ее зависимости от уровня бокового обжатия $[7,8]$. Вопрос о влиянии бокового обжатия на разгрузку представляет особый интерес, поскольку, согласно современным представлениям [19], скорость регенерации костной ткани (и, следовательно, скорость сращивания костей) зависит от действующих нагрузок (или, в общем случае, от программы нагружения).

\section{ЭКСПЕРИМЕНт}

На базе протезно-ортопедического отделения Федерального государственного унитарного предприятия «Центральный научно-исследовательский институт травматологии и ортопедии им. Н.Н. Приорова» Минздрава Российской Федерации были проведены экспериментальные измерения in vivo степени разгрузки нижней конечности при применении туторов и ее зависимости от типа и размеров ортеза, 
а также степени бокового обжатия (измеряемого на качественном уровне: без боковой компрессии; с компрессией; с большой компрессией). Тутор - это ортез, плотно охватывающий сегмент или несколько сегментов конечности, состоящий из гильз (иногда и башмачка), соединенных шинами без шарниров, с креплениями (отметим, что такие конструкции выделяют как отдельный тип в России и некоторых других странах; в англоязычной литературе подобного выделения нет и используются более общие названия, такие как ортезы, брейсы и т.п.). Гильза может быть составлена, например, из двух полуцилиндров на липучках (иногда пряжках или иных креплениях).

Измерения проводились при участии здоровых людей с неповрежденными конечностями. В эксперименте были использованы два индивидуально изготовленных под двух разных людей тутора: тутор на голень (рис. 1, a), т.е. от пальцев стопы до коленного сустава, у которого в верхней части было поставлено жесткое стальное крепление на винтах, и тутор на всю ногу с разгрузкой под тубер со стандартными креплениями на липучках (рис. 1, б). У последнего гильза тутора состоит из двух половин: задней, начинающейся от пальцев стопы и до ягодичной складки со специальной площадкой для упора в область седалищного бугра (не видна на рис. 1, б), и передней (крышка) - от головок плюсневых костей до паховой складки (показана на рис. 1, б). Нижнюю часть задней половины гильзы выполняют в виде стельки, точно соответствующей сводам стопы больного. Такое построение обеспечивает правильную опору всей подошвы на горизонтальную плоскость и одновременно сохраняет правильное соотношение оси конечности к стопе при шаговой нагрузке. С помощью липучек и/или изменения положения стальных скоб изменялся натяг (обжатие) гильзы тутора и замерялась соответствующая этому натягу нагрузка под стопой при стоянии или ходьбе.

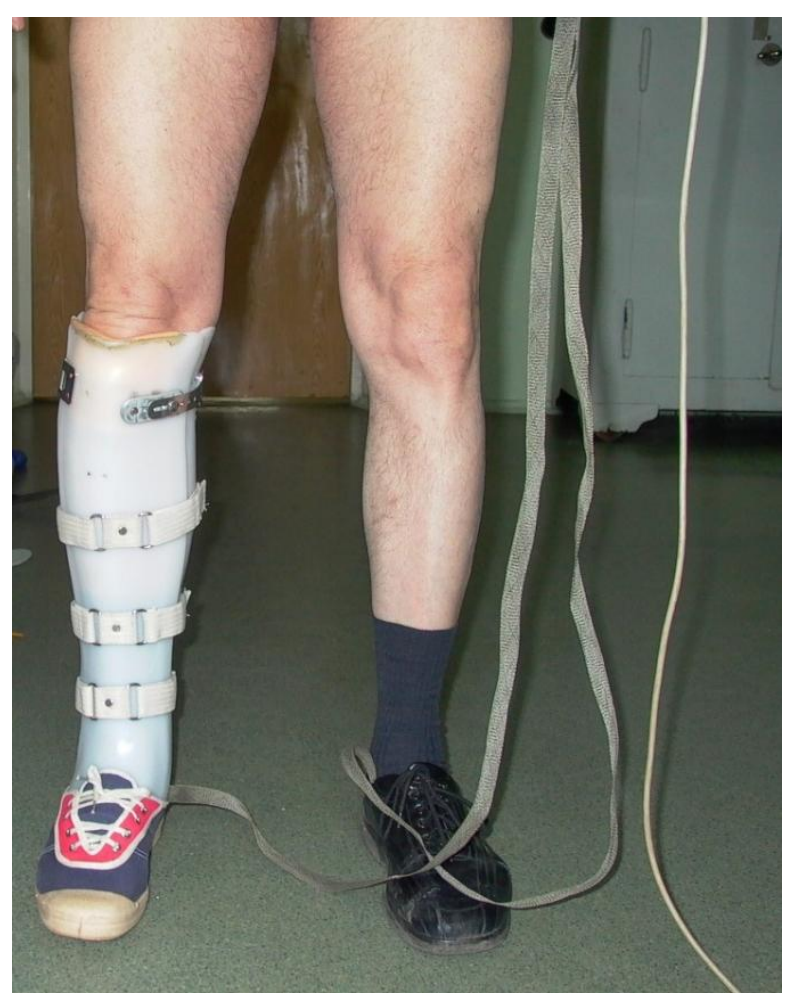

$a$

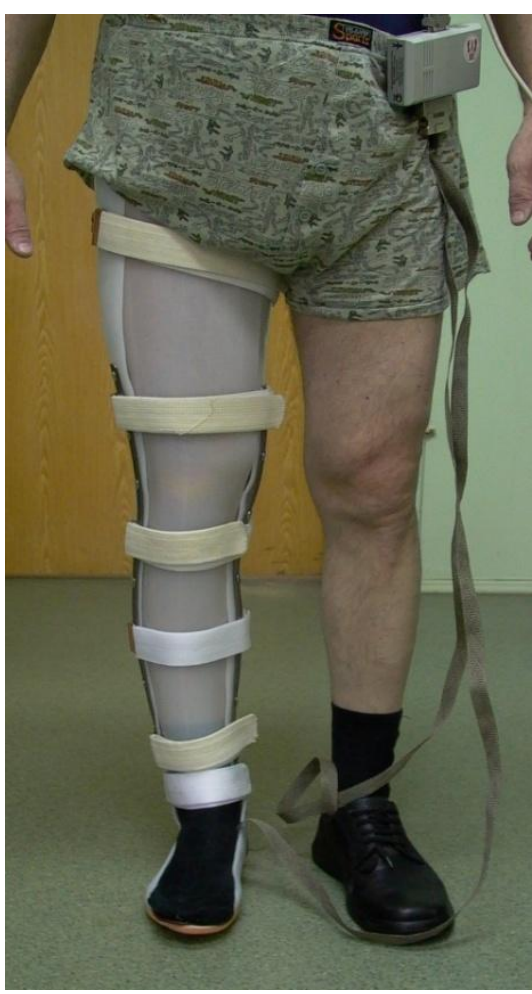

6

Рис. 1. Внешний вид испытуемых в ортезах-туторах с установленными датчиками системы «ДиаСлед»: $a$ - тутор на голень; $\sigma$ - тутор на всю ногу с разгрузкой под тубер 
Использованные туторы были изготовлены методом вакуумной формовки из полиэтилена высокого давления по слепку, снятому с ноги пациента. Для смягчения гильз ортезов в наиболее нагруженных местах: для тутора на голень - в опорной зоне связки надколенника (под коленной чашечкой), а для тутора на всю ногу - в области тубера (на чем сидим) - были применены мягкие подкладки из тонких кусков вспененного полиэтилена, приваренных к внутренней поверхности ортеза.

Измерение давления стоп на опору проводилось с помощью программноаппаратного комплекса «ДиаСлед» с использованием специальных тензометрических стелек, вкладываемых одновременно под обе стопы: для правой (ортезированной) ноги внутрь ортеза, для левой ноги - прямо в обувь (таким образом на ортезированной ноге тензометрическая стелька оказывается между стопой и подошвой ортеза - см. рис. 1, б). Комплекс предназначен для регистрации и анализа биомеханических характеристик локомоций человека путем накопления и обработки данных о распределении давления под стопами в статике и динамике в реальном масштабе времени. Установка «ДиаСлед» включает набор тензометрических стелек разных размеров с тензодатчиками в количестве двухсот на каждой, сигналы от которых после преобразования и оцифровки позволяют получить, запомнить и вывести на монитор компьютера картины распределения давлений под каждой стопой, интегральные усилия на стопу и т.П. в статике и динамике. Результаты измерений в системе «ДиаСлед» представляются в разных вариантах в нескольких полях основного окна программы (рис. 2, 3).

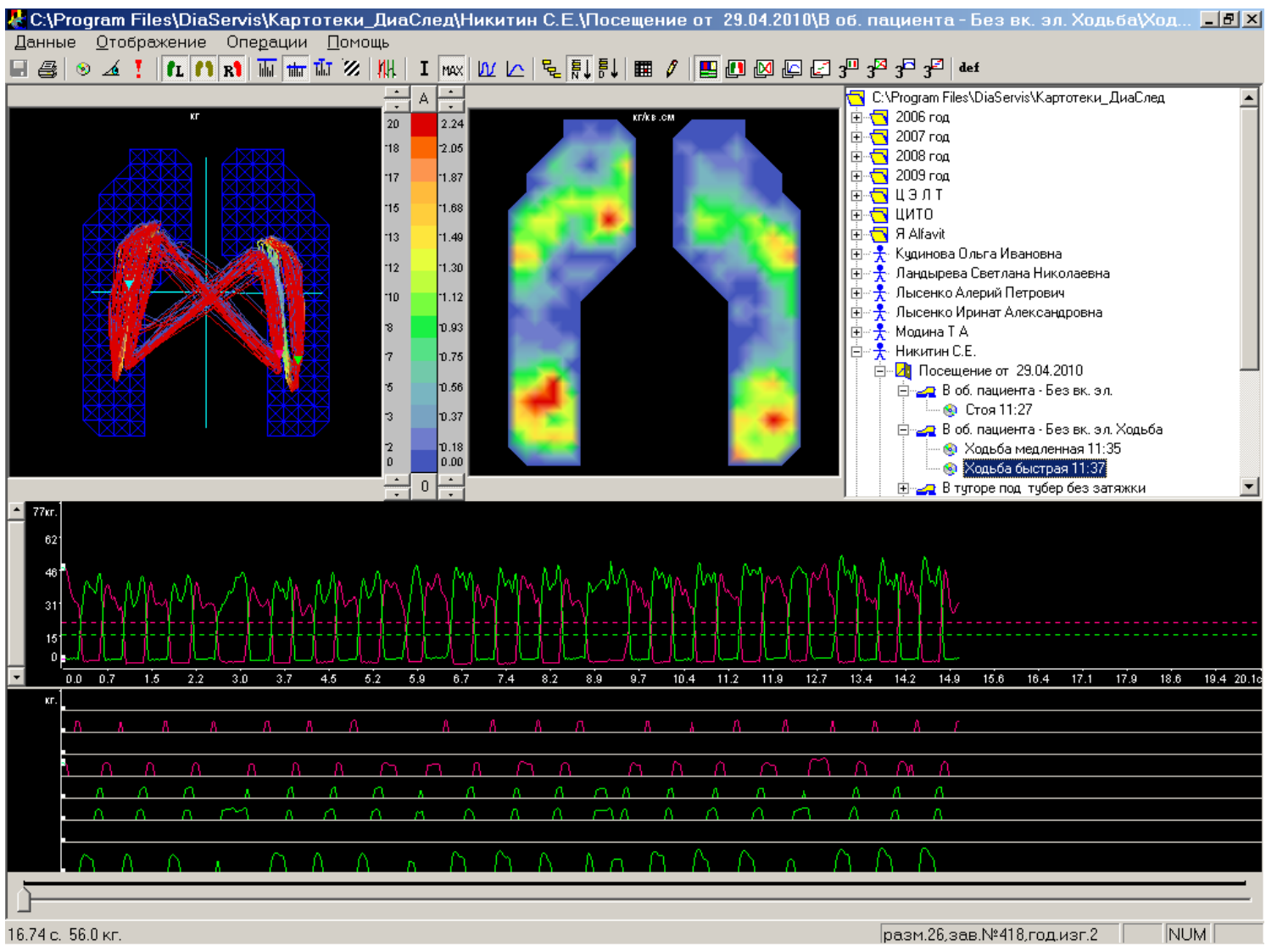

Рис. 2. Пример записи показателей в системе «ДиаСлед» здорового человека при ходьбе в обычной обуви. Асимметрия графиков интегральной нагрузки указывает на наличие систематической методической погрешности 


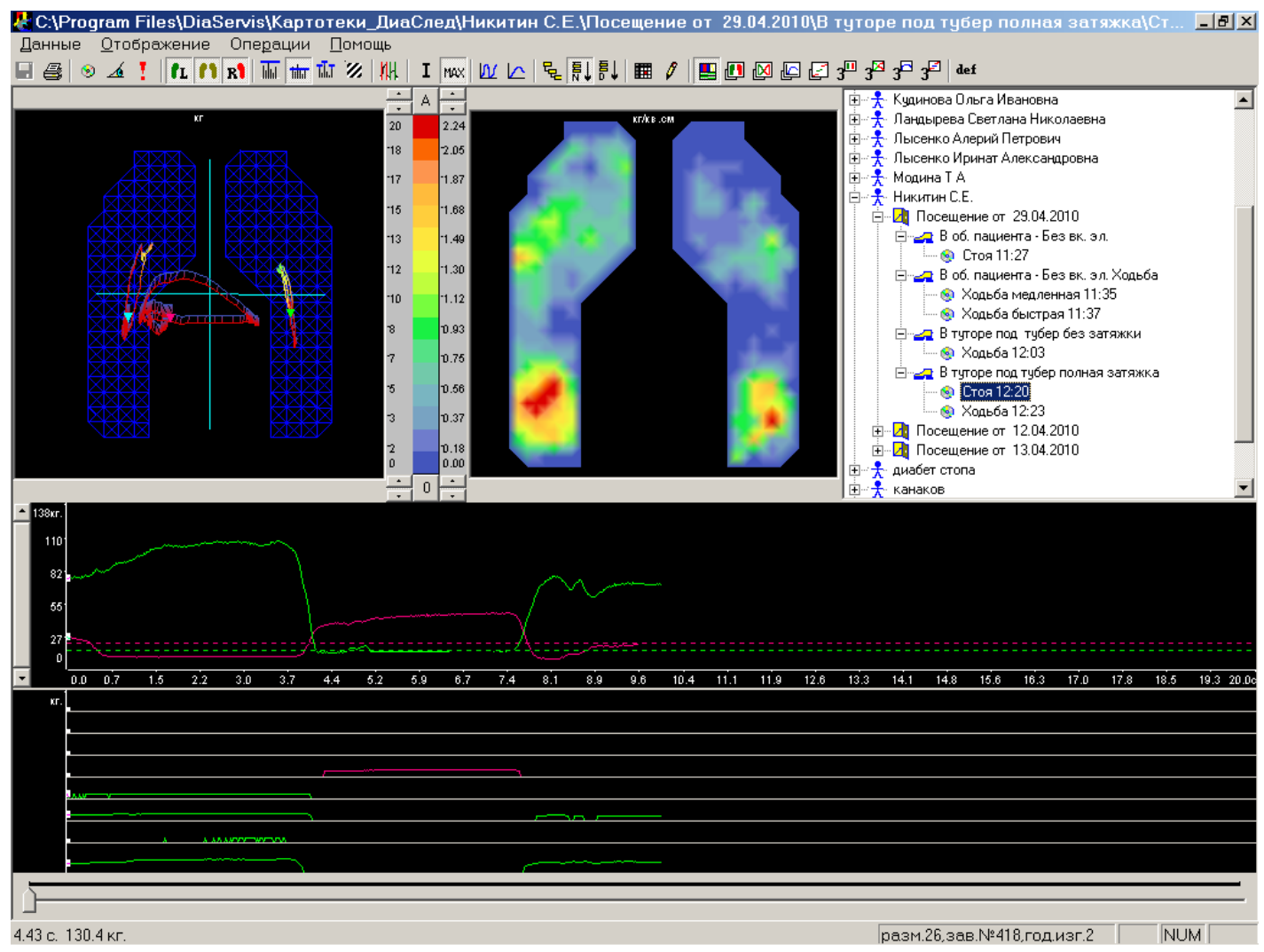

Рис. 3. Пример записи графика интегральной нагрузки стопы на опору при попеременном стоянии на одной ноге: первая фаза - опора на ногу в обуви пациента, вторая фаза - опора на ногу в туторе. Испытуемый в ортезе, большая

боковая компрессия, коэффициент разгрузки $k \approx 2,3$ (без учета погрешности)

В правом верхнем поле дан каталог пациентов, посещений, обследований и измерений, в левом верхнем поле отображаются траектории центра давления под каждой стопой и общего для обеих стоп (на рис. 2 - при ходьбе, на рис. 3 - стоя поочередно на каждой ноге); правее - топология (распределение) давлений под стопами; ниже - графики интегральной нагрузки на стопы (зеленым - на левую, красным - на правую и голубым - на обе стопы); внизу окна - подограмма, т.е. графики нагрузки на различные участки стопы: пятку, область свода, головки плюсневых костей, пальцы. На графиках интегральной нагрузки (с которыми мы более всего и работали) по оси абсцисс откладывается время в секундах, по оси ординат - суммарная нагрузка на каждую из стоп. При этом указываемые на графиках единицы измерения кгс для численных значений нагрузки на самом деле условны и не дают реальных значений в кгс, а подлежат дополнительной обработке и нормировке, после чего могут быть достоверно использованы как относительные показатели.

Для измерения в положении стоя траектории центра давления выглядят в виде точек или почти прямых дуг, а все графики - в виде отрезков прямых (см. рис. 3). При ходьбе траектория общего центра давления для обеих стоп превращается в «бабочку», а графики интегральной нагрузки приобретают циклический характер (см. рис. 2). В норме все характеристики для обеих стоп должны быть идентичны, а картинки симметричны, при патологии и/или использовании ортезов симметрия нарушается.

Для оценки степени разгрузки конечности при ортезировании были проведены исследования в различных режимах. В начале обследования были записаны параметры стояния и ходьбы испытуемых в обычной повседневной обуви (см. рис. 2). После этого записывались как статические (см. рис. 3), так и динамические (см. рис. 2, 4) 
показатели изменения давления стопы на тензометрическую стельку в ортезах при разной степени обжатия конечности внутри изделия (фиксирующие ремни ортеза затягивались на разную величину). Поскольку в клинике пациенты часто боятся опираться на больную ногу, то испытуемым предъявлялось требование осуществления ходьбы, максимально приближенной к норме, т.е. необходимо было стараться достигать полной опоры на каждую из конечностей, при этом ходьба в ортезе осуществлялась без дополнительной опоры (костылей, трости и т.п.). Для достижения максимально полной весовой нагрузки ее измерения производились не только в момент ходьбы, но и в момент стояния на одной ноге.

Из рис. 2 и 4 (верхний график) видно, что при измерениях показателей здоровых испытуемых в обычной обуви (без ортезов) снимаемые диаграммы получились несимметричными. Это свидетельствует о систематической методической погрешности, причину которой установить не удалось, но которая тем не менее должна быть учтена при обработке полученных данных. Согласно приведенным графикам она составляет примерно $20 \%$. Анализ результатов экспериментального исследования с поправкой на указанную систематическую погрешность позволил определить степень разгрузки конечности внутри тутора (табл. 1). Коэффициент разгрузки $k$ рассчитывался из графиков интегральной нагрузки (стоя или по всему единичному измерению при ходьбе) как отношение максимальной нагрузки на неортезированную конечность (а в нашем случае это всегда была левая нога) к максимальной нагрузке на конечность в ортезе (см. пример на рис. 4 с коэффициентом разгрузки $k \approx 1,3$ без учета погрешности). Показатели разгрузки можно вводить и по-другому. Например, в [39] используется индекс, показывающий на сколько процентов уменьшается нагрузка

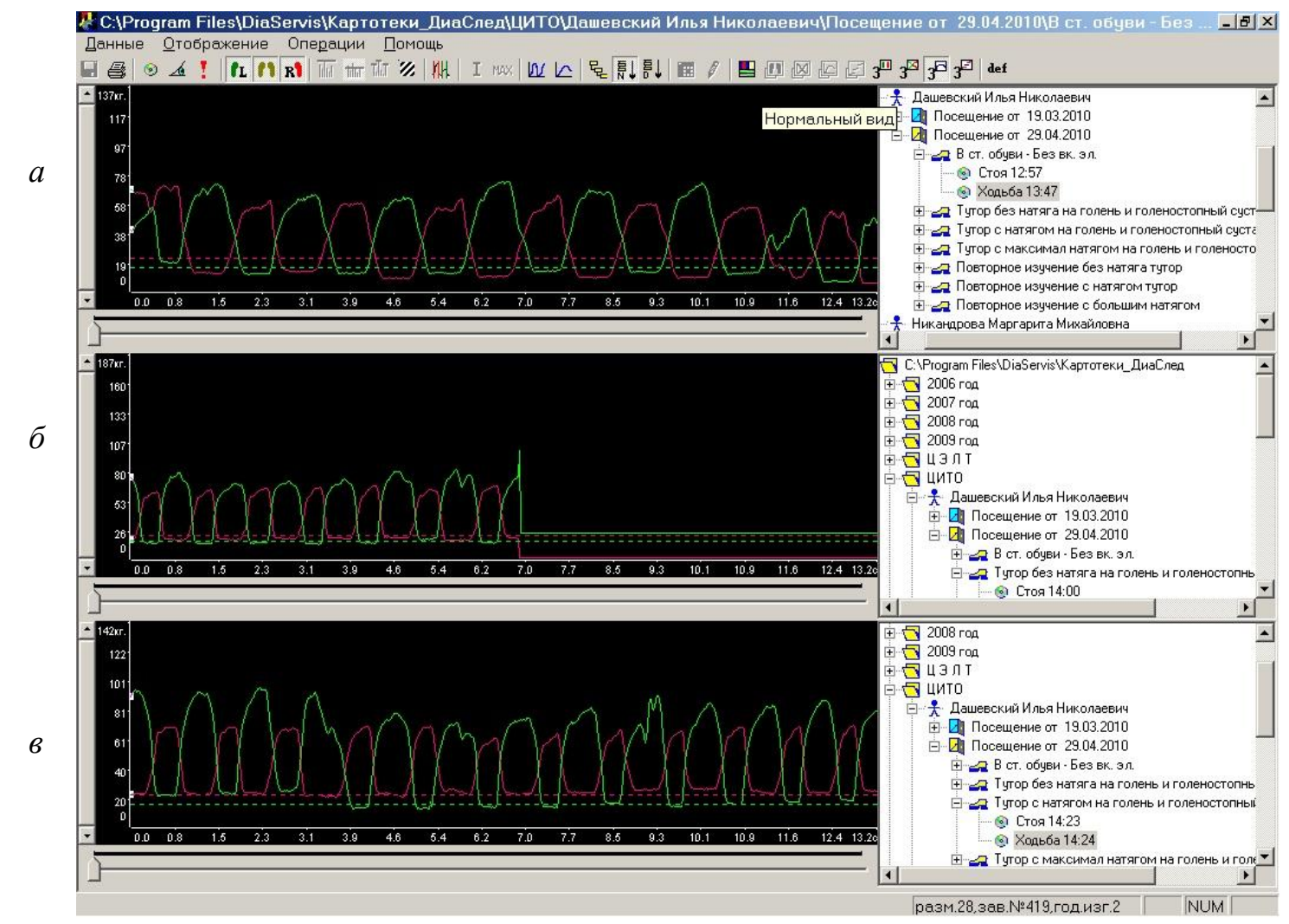

Рис. 4. Графики интегральной нагрузки при ходьбе (яркая кривая - на конечность в обуви, бледная кривая - на конечность в туторе): $a$ - в обуви пациента; $\sigma$ - в туторе без дополнительного обжатия; в - в туторе при средней степени обжатия при $k=1,3$ 
на конечность при ее ортезировании разными ортезами. Оба индекса легко пересчитываются друг в друга. Наблюдаемый в табл. 1 разброс результатов связан как с ограниченной точностью системы «ДиаСлед», так и с некоторой недоработанностью использованной методики, которую предполагается в дальнейшем усовершенствовать для достижения большей стабильности, точности и достоверности получаемых результатов. Как видно из табл. 1, значения коэффициентов разгрузки располагаются в диапазоне примерно от 1 до 2,4.

Таблица 1

Значения коэффициента разгрузки в эксперименте

\begin{tabular}{|c|c|c|c|c|c|c|c|}
\hline Эксперимент & $\begin{array}{c}\text { В поло- } \\
\text { жении } \\
\text { стоя (1) }\end{array}$ & $\begin{array}{c}\text { В поло- } \\
\text { жении } \\
\text { стоя (2) }\end{array}$ & $\begin{array}{c}\text { В поло- } \\
\text { жении } \\
\text { стоя (3) }\end{array}$ & $\begin{array}{c}\text { В поло- } \\
\text { жении } \\
\text { стоя (4) }\end{array}$ & $\begin{array}{c}\text { При } \\
\text { ходьбе } \\
(1)\end{array}$ & $\begin{array}{c}\text { При } \\
\text { ходьбе } \\
(2)\end{array}$ & $\begin{array}{c}\text { Продьбе } \\
\text { (3) }\end{array}$ \\
\hline В обуви пациента & 1 & - & - & 1 & - & 1 & 1 \\
\hline $\begin{array}{c}\text { В ортезе } \\
\text { без боковой } \\
\text { компрессии }\end{array}$ & 1,6 & 2,4 & 1,4 & - & 1,1 & 1 & $<1 ?$ \\
\hline $\begin{array}{c}\text { В ортезе } \\
\text { с боковой } \\
\text { компрессией }\end{array}$ & 1,1 & 1,2 & 1,1 & - & 1,0 & 1,1 & - \\
\hline $\begin{array}{c}\text { В ортезе с } \\
\text { большой боковой } \\
\text { компрессией }\end{array}$ & - & 1,4 & 1,2 & 1,8 & 1,1 & - & 1,4 \\
\hline
\end{tabular}

Примечание: курсивом в строке заголовка выделены данные, относящиеся к ортезу на всю ногу, остальные данные - для ортеза на голень.

\section{БИОМЕХАНИЧЕСКАЯ МОДЕЛЬ РАЗГРУЗКИ КОНЕЧНОСТИ В ТУТОРЕ}

В норме вес тела передается через трубчатые кости конечности и хрящевые диски на арку стопы, через нее (на первой фазе шага) на пятку, подпяточный слой мягких тканей и наконец на поверхность опоры. Эффект разгрузки при наложении ортеза связан, очевидно, с тем, что часть веса тела снимается с трубчатых костей, переносится на корпус ортеза и передается на опору через него. Причина возникновения эффекта разгрузки при ортезировании нижних конечностей в общем виде представляется нам следующей. Слепок-негатив, по которому затем изготавливается ортез, снимается с ненагруженной конечности, находящейся в горизонтальном положении. При переходе человека из положения сидя/лежа в положение стоя длина его нижних конечностей под действием веса тела стремится к сокращению, чему препятствует плотно подогнанный к конечности ортез, на который и переносится при этом часть веса тела.

Можно указать следующие механизмы разгрузки конечности в туторе:

1) Наличие зон локальных опор на мыщелки, связку надколенника и бугор седалищной кости, принимающих на себя часть веса тела.

2) Разгрузка на поверхность гильзы ортеза через слой мышечных тканей за счет трения/сцепления.

3) «Эффект воронки», заключающийся в передаче части нагрузки на коническую часть гильзы ортеза: голень находится в изделии как в воронке, в которую она при действии осевой нагрузки может слегка просесть, но не может полностью провалиться. 
Боковое обжатие ортеза (липучками, ремешками и т.п.), по-видимому, должно усиливать эти эффекты.

Выделим следующие основные несущие (силовые) элементы биомеханической модели нога-ортез: трубчатые кости, хрящевые диски, арка стопы (вместе с пяткой и подподошвенным слоем мягких тканей), слой мышечных тканей (например, для голени - это икроножная, камбаловидная и др. мышцы), гильза ортеза, локальные опоры (рис. 5, a).

Для уяснения качественных особенностей механизмов разгрузки и получения оценок для конструктивных жесткостей основных элементов системы нога-ортез начнем с аппроксимации сложного реологического поведения биологических тканей [32] упругими линейными и изотропными зависимостями [5] с использованием модуля Юнга и коэффициента Пуассона, как это нередко и делается на практике.

Ортез схематизируем тонкостенным цилиндром, трубчатую кость и мышцы голени - толстостенными полыми цилиндрами, хрящи - дисками, локальные опоры параллелепипедами. Используем индексы $o, b, l, m, c$ как метки соответственно ортеза (orthosis), кости (bone), локальных опор (local), мышечных тканей (muscle), хряща (cartilage) и введем обозначения: $E$ - модуль Юнга, $L$ - длина трубчатой кости, ортеза, мышц голени, $S$ - площадь поперечного сечения или опоры, $D_{o}$ и $t_{o}-$ диаметр и толщина стенки ортеза, $d_{b}$ и $D_{b}-$ внутренний и внешний диаметры трубчатой кости, $H_{l}$, $h_{l}, t_{l}$ - габариты (длина, ширина и толщина) локальной опоры (из вспененного полиэтилена), $\sigma$ - напряжение, $\varepsilon$ - деформация, $P$ - действующие усилия, $\delta$ - перемещение (укорочение, сжатие), $F$ - конструктивная жесткость, $F \equiv P / \delta$.

Примерные механические и геометрические характеристики ортеза, локальных опор, костей, мышечных тканей и хрящевого диска по результатам непосредственного обмера ортезов и с использованием данных «ЦИТО» и других источников $[2-5,9,12$, $21,23,25,26,29,33,35,36]$ даны в табл. 2.

Приведенные данные позволяют дать оценки по порядку величины конструктивных жесткостей $F$ некоторых основных элементов системы нога-ортез: гильзы ортеза, трубчатых костей, локальных опор и хрящевого диска.

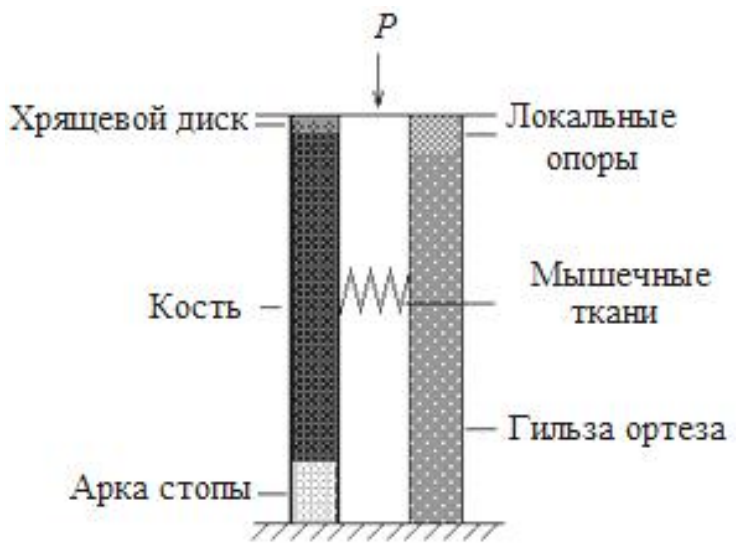

$a$

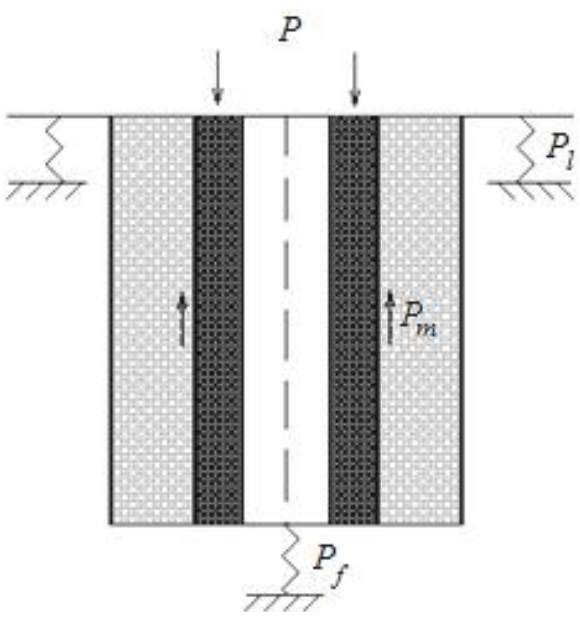

б

Рис. 5. Силовые элементы (a) и биомеханическая модель (б) системы нога-ортез $\left(P\right.$ - вес тела, $P_{l}$ - часть веса тела, принимаемая локальными опорами; $P_{m}$ - часть веса, передаваемая на поверхность гильзы ортеза через слой мышечных тканей; $P_{f}$ - часть веса, передаваемая на подошву ортеза через трубчатую кость) 
Механические и геометрические характеристики основных элементов системы нога-ортез

\begin{tabular}{|c|c|c|c|c|c|c|}
\hline Обозначение & $E$, Па & $L, \mathrm{~m}$ & $D, \mathrm{M}$ & $d, \mathrm{M}$ & $H, h, \mathrm{M}$ & $t, \mathrm{M}$ \\
\hline Ортез, $o$ & $5,5 \cdot 10^{8}$ & $5 \cdot 10^{-1}$ & $10^{-1}$ & - & - & $5 \cdot 10^{-3}$ \\
\hline Кость, $b$ & $1,8 \cdot 10^{10}$ & $5 \cdot 10^{-1}$ & $4 \cdot 10^{-2}$ & $2 \cdot 10^{-2}$ & - & - \\
\hline $\begin{array}{c}\text { Локальные } \\
\text { опоры, } l\end{array}$ & $0,5 \cdot 10^{6}$ & - & - & - & $\begin{array}{c}5 \cdot 10^{-2} \\
2 \cdot 10^{-2}\end{array}$ & $10^{-2}$ \\
\hline $\begin{array}{c}\text { Мышечные } \\
\text { ткани, } m\end{array}$ & $G \approx(7,7 \div 24,3) 10^{3}[26]$ & $5 \cdot 10^{-1}$ & $10^{-1}$ & $4 \cdot 10^{-2}$ & - & - \\
\hline Хрящ, $c$ & $10^{7}$ & - & $5 \cdot 10^{-2}$ & - & - & $2 \cdot 10^{-3}$ \\
\hline
\end{tabular}

К примеру, для гильзы ортеза (тонкостенный цилиндр)

$$
F_{o}=\frac{P}{\delta} \sim \frac{\sigma_{o} S_{o}}{L_{o} \varepsilon_{o}} \sim\left|\frac{\sigma}{\varepsilon} \sim E\right| \sim \frac{\pi D_{o} t_{o} E_{o}}{L_{o}} \sim 1,7 \cdot 10^{6} \frac{\mathrm{H}}{\mathrm{M}} .
$$

Поскольку при ортезировании подошва ортеза в точности моделирует свод стопы, то при наложении ортеза фактически арка стопы, подошвенный слой мягких тканей и подошва ортеза при нагружении работают совместно, как единая конструкция в виде «сэндвича». Учитывая, что сколько-нибудь точный теоретический расчет податливости такого «сэндвича» представляет значительные трудности, мы оценили ее прямым измерением укорочения конечности в ортезе под действием веса тела при переходе из положения лежа/сидя в положение стоя (рис. 6).

При весе 83 кгс осадка составила порядка 7 мм, давая таким образом для конструктивной жесткости сэндвича стоп

$$
F_{f}=\frac{P}{\delta} \sim \frac{83 \text { кгс }}{7 \mathrm{MM}} \approx 0,12 \cdot 10^{6} \frac{\mathrm{H}}{\mathrm{M}} .
$$
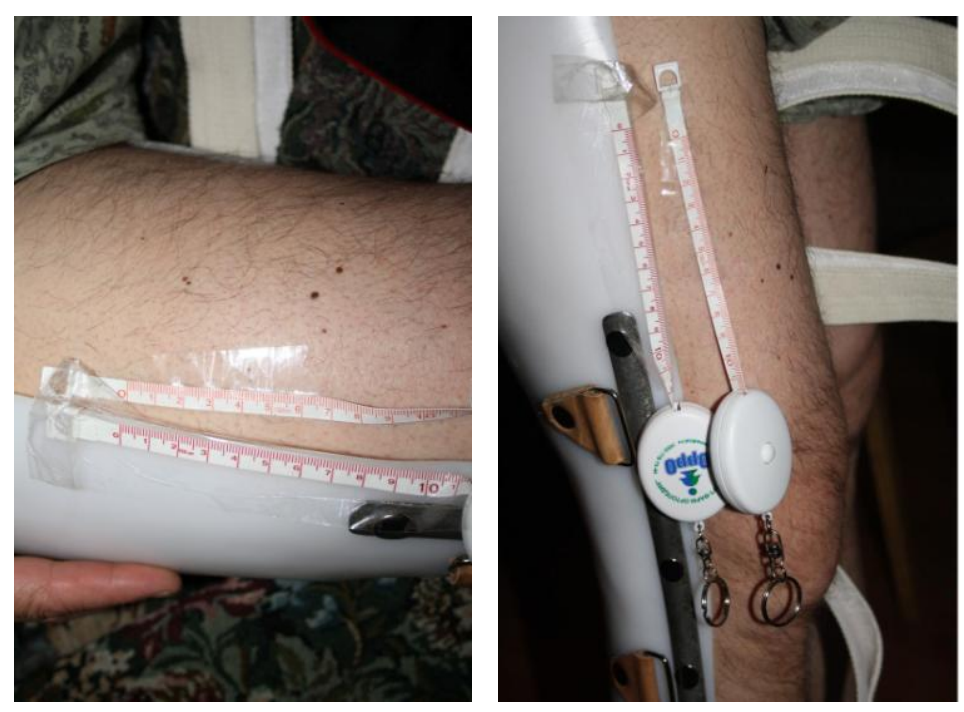

Рис. 6. Экспериментальная оценка жесткости «сэндвича» стопы 
Согласно сделанным при проведении экспериментов наблюдениям, при переходе в вертикальное положение проскальзывания конечности в туторе не происходит, и таким образом силы трения между ногой и тутором работают по механизму полного сцепления. Это означает, что поверхность голени сцеплена с ортезом, а осадка возникает за счет проседания центральной, осевой части голени. Мы принимаем для расчета осадки следующую схему: внутри жесткой цилиндрической обоймы (ортеза) находится жесткий цилиндр (кость), нагруженный весом тела и подвешенный на окружающем его слое мышечных тканей (который мы для простоты схематизируем полым цилиндром), снаружи сцепленным с ортезом.

Оценка жесткости такой конструкции:

$$
\begin{gathered}
F_{m}=\frac{P}{\delta} \sim \frac{\tau_{m} S_{m}}{t_{m} \gamma_{m}} \sim\left|\frac{\tau}{\gamma} \sim G\right| \sim G_{m} \frac{2 \pi R_{o} L_{o}}{R_{o}-R_{b}}=G_{m} \frac{2 \pi D_{o} L_{o}}{D_{o}-D_{b}} \sim \\
\sim \frac{2 \cdot 3,14 \cdot 10^{-1} \mathrm{M} \cdot 5 \cdot 10^{-1} \mathrm{M}(7,7 \ldots 24,3) 10^{3} \Pi \mathrm{a}}{(10-4) 10^{-2} \mathrm{M}} \approx(0,04 \ldots 0,13) 10^{6} \frac{\mathrm{H}}{\mathrm{M}},
\end{gathered}
$$

где $\tau$ - касательное напряжение, $\gamma$ - сдвиговая деформация (угол сдвига); $G$ - модуль сдвига мягких тканей конечности.

Чуть изящнее те же соображения можно формализовать на основе осесимметричной антиплоской задачи [22] о провисании двухслойного цилиндра с жестким ядром на некоторую величину $\delta$ под действием заданного веса $P$ или задачи об осевом сдвиге полого кругового цилиндра. При этом отличными от нуля оказываются лишь две компоненты напряженно-деформированного состояния: касательное напряжение $\tau_{r 3} \equiv \tau$ и вертикальная компонента перемещений $u(r)$, являющаяся гармонической функцией, где $r$ - полярный радиус. Математически задача приводится к краевой задаче Дирихле для уравнения Лапласа относительно $u(r)$ в кольцевой области

$$
\Delta u=0, u\left(R_{o}\right)=0, u\left(R_{b}\right)=\delta,
$$

где $R_{o}, R_{b}$ - радиусы соответственно ортеза и кости, и положительное направление для перемещений $u$ выбрано вниз.

Эту задачу можно легко решить, расписывая в (2) осесимметричный лапласиан $\Delta$ в полярных координатах [8]. Однако более простым и прямо ведущим к цели представляется решение задачи в напряжениях с последующим применением теоремы Кастильяно. Действительно, рассматриваемая задача оказывается статически определимой.

Как следствие, единственная ненулевая компонента тензора напряжений $\tau_{r z}$ легко находится из условия равновесия цилиндра, получаемого путем сечения исходного цилиндра коаксиальной ему цилиндрической поверхностью промежуточного радиуса $r\left(R_{b}<r<R_{o}\right)$ и последующего отбрасывания внешней части

$$
P=\tau_{r z}(r) 2 \pi r \cdot L, \quad \tau_{r z}(r)=\frac{P}{2 \pi r \cdot L},
$$

где $P$ - вес тела, передаваемый через трубчатые кости; $L$ - длина ортеза.

Плотность упругой энергии $w$ выражается через напряжения формулой [28] 


$$
\begin{gathered}
w=\frac{1}{2 E}\left[\sigma_{x}^{2}+\sigma_{y}^{2}+\sigma_{z}^{2}-2 \mu\left(\sigma_{y} \sigma_{z}+\sigma_{z} \sigma_{x}+\sigma_{x} \sigma_{y}\right)\right]+\frac{1}{2 G}\left(\tau_{y z}^{2}+\tau_{z x}^{2}+\tau_{x y}^{2}\right)= \\
=\frac{\tau_{r z}^{2}}{2 G}=\frac{1}{2 G}\left(\frac{P}{2 \pi L}\right)^{2} \frac{1}{r^{2}},
\end{gathered}
$$

где $G$ - модуль сдвига.

Для линейно-упругого тела дополнительная работа равна энергии деформации, которая находится интегрированием плотности упругой энергии по объему цилиндра, занимаемого мышечным слоем:

$$
W=L \cdot 2 \pi \frac{1}{2 G}\left(\frac{P}{2 \pi L}\right)^{2} \int_{R_{b}}^{R_{o}} \frac{r d r}{r^{2}}=\frac{P^{2}}{4 \pi L G} \ln \frac{R_{o}}{R_{b}}=W(P) .
$$

По теореме Кастильяно соответствующее обобщенной силе $P$ обобщенное перемещение $\delta$ (осадка конечности под действием веса тела $P$ ) равно

$$
\delta=\frac{\partial W}{\partial P}=\frac{P}{2 \pi L G} \ln \frac{R_{o}}{R_{b}},
$$

и для жесткости как коэффициента пропорциональности между $P$ и $\delta$ получаем

$$
F_{m}=\frac{2 \pi G L}{\ln \frac{R_{o}}{R_{b}}} \cong \frac{2 \cdot 3,14(7,7 \ldots 24,3) 10^{3} \Pi \mathrm{a} \cdot 5 \cdot 10^{-1} \mathrm{M}}{\ln \left(\frac{5}{2}\right)} \approx(0,026 \ldots 0,083) 10^{3} \frac{\mathrm{H}}{\mathrm{M}} .
$$

Полученная формула идентична (1) с точностью до замены коэффициента

$$
\frac{R_{o}}{R_{o}-R_{b}} \sim \frac{5}{5-2}=\frac{5}{3} \quad \text { на } \frac{1}{\ln \frac{R_{o}}{R_{b}}} \sim \frac{1}{\ln \frac{5}{2}}=\frac{1}{\ln (2,5)} \sim \frac{1}{0,92}
$$

с уменьшением жесткости примерно в $5 \cdot 0,92 / 3$, т.е. примерно в 1,5 раза. Полученные оценки для жесткостей $F, 10^{6} \mathrm{H} / \mathrm{M}$, таковы: трубчатые кости $b-40$; хрящи $c-10$; «сэндвич» стопы $f-0,12$; гильза ортеза $o-1,7$; локальные опоры $l-0,05$; мышечная подвеска $m-0,026 \ldots 0,083$.

Сравнение жесткостей, работающих в одной последовательной силовой цепи кости, хряща и «сэндвича» стопы, показывает, что кость и хрящ можно считать абсолютно жесткими и учитывать лишь податливость «сэндвича» стопы. Аналогично можно пренебречь податливостью гильзы ортеза в цепи «локальные опоры-ортез». Таким образом, биомеханику разгрузки в системе нога-тутор можно формализовать в виде схемы (рис. 5, б). Здесь $P_{f}$ - нагрузка, приходящаяся на стопу - это и есть остаточная нагрузка на конечность (на «сэндвич» стопы). Именно ее мы и измеряем тензометрическими стельками в эксперименте и для нее же стремимся получить расчетные оценки на основе биомеханической модели.

С точки зрения механики, представленная схема соответствует статически неопределимой задаче о грузе весом $P$, поддерживаемом тремя опорами разной жесткости. Это стандартная задача сопротивления материалов, решение которой методом сил [28] дает 


$$
P_{f}=F_{f} w=\frac{F_{f} P}{F_{f}+F_{l}+F_{m}}=\frac{P}{1+\frac{F_{l}}{F_{f}}+\frac{F_{m}}{F_{f}}}=\frac{P}{1+k_{l}+k_{m}}=\frac{P}{k}, \quad k=1+k_{l}+k_{m},
$$

где

$k_{l}=\frac{F_{l}}{F_{f}} \sim \frac{0,05}{0,12} \approx 0,4, \quad k_{m}=\frac{F_{m}}{F_{f}} \sim \frac{0,026 \ldots 0,83}{0,12} \sim 0,2 \ldots 0,7, \quad k \sim 1+0,4+(0,2 \ldots 0,7)=$ $=1,6 \ldots 2,1 ; k, k_{l}, k_{m}$ - частотные коэффициенты разгрузки.

Введенный здесь коэффициент $k$ как раз и представляет по своему смыслу интересующий нас коэффициент разгрузки конечности в ортезе и показывает, во сколько раз уменьшается нагрузка на конечность при наложении ортеза. Коэффициенты $k_{l}$ и $k_{m}$ отвечают вкладу в разгрузку за счет локальных опор и обоймы ортеза.

Модель, представленная схемой на рис. 5, 6, не учитывает зависимости разгрузки от степени боковой компрессии. Такая зависимость может быть связана с «эффектом воронки» (конусности) и так называемым «поршневым эффектом» (растяжением кожи под нагрузкой, а также ее сдвигом-проскальзыванием по тонкому и мягкому слою подкожной жировой клетчатки). Нельзя исключать и возможность появления в каких-то случаях эффекта проскальзывания между ногой и гильзой ортеза. Эти эффекты в совокупности могут приводить к выталкиванию конечности вверх с ростом бокового обжатия.

Указанные эффекты можно в грубом приближении учесть с помощью модели гладкого жесткого конуса (конечность), скользящего вверх при сжатии конической обоймы (подтяжка гильзы ортеза) (рис. 7,a). Данные на рис. 5, 6 начинают соответствовать данным рис. 7, б, где аналогично рис. 5, 6 получим

$$
\frac{P_{f}}{P}=\frac{1}{1+k_{l}+k_{m}}\left(1-\frac{F_{m}}{2 \pi \operatorname{tg} \alpha \cdot P} \Delta l\right), \quad P_{f}=\frac{P}{\frac{1+k_{l}+k_{m}}{\left(1-\frac{F_{m}}{2 \pi \operatorname{tg} \alpha \cdot P} \Delta l\right)}},
$$

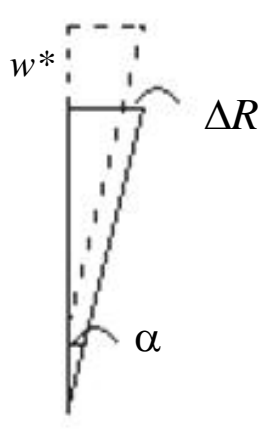

$a$

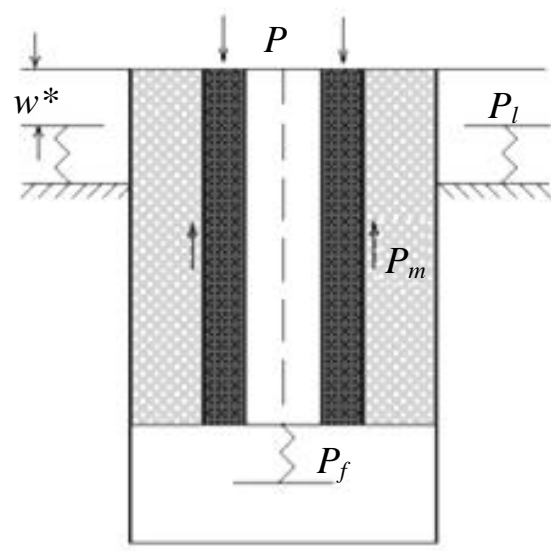

6

Рис. 7. Учет в модели зависимости разгрузки от степени боковой компрессии: $a$ - модели гладкого жесткого конуса (конечность), скользящего вверх при сжатии конической обоймы (подтяжка гильзы ортеза); б - биомеханическая модель системы нога-ортез ( $P$ - вес тела, $P_{l}$ - часть веса тела, принимаемая локальными опорами; $P_{m}$ - часть веса, передаваемая на поверхность гильзы ортеза через слой мышечных тканей; $P_{f}-$ часть веса, передаваемая на подошву ортеза через трубчатую кость) 
где $\alpha$ - угол конусности конечности $\left(\alpha \sim 15^{\circ}=\pi / 12\right)$; на рис. $7, a \Delta R-$ уменьшение радиуса гильзы ортеза при окружной подтяжке крепления (липучек, пряжек и т.п.) на $\Delta l=2 \pi \Delta R ; w^{*}-$ возникающее при этом выталкивание конечности вверх; $P$ - вес тела, $P \sim 100$ кгс $\sim 10^{3}$ Н. Нагрузка $P_{f}$ на конечность с увеличением подтяжки $\Delta l$ падает, а знаменатель последней дроби, который как раз и представляет собой коэффициент разгрузки, растет с ростом $\Delta l$. Полной разгрузке конечности $P_{f}=0$, при которой стопа внутри ортеза как бы перестает касаться опоры, отвечает условие

$$
P-F_{m} \frac{\Delta L}{2 \pi \operatorname{tg} \alpha}=0, \Delta L=2 \pi \cdot \operatorname{tg} \alpha \frac{P}{F_{m}} \sim(17 \ldots 1,7) \mathrm{cm} .
$$

\section{ОБСУЖДЕНИЕ}

Расчетное значение коэффициента разгрузки $k \approx 1,6 \ldots 2,1$ согласуется c результатами эксперимента. Это свидетельствует о качественной адекватности модели и дает надежду на то, что развитие использованных подходов позволит добиться лучшего количественного соответствия результатов. В то же время разброс экспериментальных данных говорит о необходимости усовершенствования методики измерений коэффициента разгрузки. Основные погрешности здесь связаны, с одной стороны, с некоторым произволом в технологии изготовления и применения ортезов. Поэтому для повышения точности и воспроизводимости измерений необходимо более четко регламентировать все этапы и аспекты процесса ортезирования от процедуры снятия слепка до надевания ортеза и манипуляций по его подтяжке (включая и контроль условий контакта конечность-ортез). Другая возможная причина несовершенство принятого алгоритма определения коэффициента разгрузки по снятым графикам интегральной нагрузки на конечности.

Один из важных практических вопросов ортезирования нижних конечностей можно ли управлять разгрузкой конечности в ортезе путем изменения боковой компрессии? Имеющиеся экспериментальные данные не дают ответа на этот вопрос, для его получения надо, как сказано выше, усовершенствовать методику измерений коэффициента разгрузки. Теория же говорит о том, что при отсутствии проскальзывания между ногой и ортезом (минимальная базовая модель) коэффициент разгрузки не зависит от натяга, в условиях проскальзывания (расширенная базовая модель) конечность при увеличении бокового обжатия будет выталкиваться вверх и коэффициент разгрузки будет расти. В реальности условия контактного взаимодействия между ногой и ортезом не соответствуют ни полному сцеплению, ни идеальному проскальзыванию - это, скорее, условия сухого трения, которые в зависимости от величин коэффициентов трения, механических и геометрических характеристик контактирующих тел, а также действующих нагрузок могут приводить к образованию площадок как сцепления, так и скольжения разного размера. Поскольку мы наблюдали отсутствие скольжения, можно полагать, что набор механикогеометрических параметров, характерных для проведенных экспериментов, приводит к полному сцеплению между ногой и ортезом, а «поршневой эффект» недостаточен для полноценной имитации скольжения. Таким образом, для теоретического анализа возможности управления разгрузкой необходимо дополнить модель учетом трения между ногой и ортезом и выяснить, при каких значениях параметров может реализовываться проскальзывание. При этом более или менее полный учет вышеперечисленных факторов возможен лишь на основе численного расчета, отдельные же закономерности, вероятно, можно выявить при исследовании простейших аналитических моделей. 
Далее, результаты анализа модели показывают, что коэффициент разгрузки существенно зависит от значений упругих модулей (как и от механической модели в целом) мягких тканей голени. Между тем характеристики эти индивидуальны, чрезвычайно вариабельны от человека к человеку и изменчивы даже у одного и того же индивида как во времени, так и в зависимости от ряда других факторов. К числу последних относится, например, уровень активации (напряжения) мышцы (по [25] поперечный модуль сдвиговой упругости $G$ для расслабленного и напряженного бицепса составляет соответственно 7,7 и 24,3 кПа - разница в разы), который сам может зависеть от характера и степени фиксации сегмента и т.П. При этом разница в значениях коэффициентов разгрузки в зависимости только лишь от уровня активации мышечных тканей при прочих равных условиях может согласно модели составлять до $30 \%$ (именно это и привело к полученному в расчетах диапазону для коэффициента разгрузки $1,6 \ldots 2,1)$. Таким образом, желательно иметь возможность одновременного прямого измерения in vivo механических характеристик мягких тканей голени, что существенно усложняет задачу. При практической же реализации идеи управления разгрузкой для оптимизации хода лечения предпочтительнее прямой контроль (а лучше мониторинг) и задание непосредственно коэффициента разгрузки.

\section{Выводы}

Оценки для коэффициента разгрузки при ортезировании нижней конечности, полученные, с одной стороны, в прямых измерениях, а с другой - при расчетах на основе предложенной биомеханической модели, оказались достаточно согласованы.

Для повышения точности эксперимента необходима более четкая регламентация процессов изготовления, наложения и подтяжки ортезов.

Анализ возможности управления разгрузкой и выяснение соответствующих диапазонов параметров требуют дополнения разработанной модели с учетом трения между ногой и ортезом.

Ввиду чрезвычайной изменчивости механических характеристик мягких тканей даже у одного и того же человека как во времени, так и в зависимости от многих параметров, для практической реализации идеи управления разгрузкой желательно иметь возможность прямого контроля и задания степени разгрузки.

\section{БЛАГОДАРНОСТИ}

Авторы признательны А.Л. Скубачевскому и Р.В. Гольдштейну, благодаря которым стала возможной эта работа, Н.М. Осипенко за полезные обсуждения, а также А.К. Цатуряну и анонимным рецензентам заявки на грант РФФИ за доброжелательную, содержательную и конструктивную критику.

Работа выполнена при частичной финансовой поддержке грантов РФФИ № 11-08-01243, 14-08-01266 и 14-08-01163.

\section{СПИСОК ЛИТЕРАТУРЫ}

1. Азизян Р.В. Комплексное лечение множественных переломов нижних конечностей с применением ортезов: автореф. дис. ... канд. мед. наук. - Ереван, 2002.

2. Бегун П.И., Шукейло Ю.А. Биомеханика: учебник для вузов. - СПб.: Политехника, 2000. - 463 с.

3. Березовский В.А., Колотилов Н.Н. Биофизические характеристики тканей человека: справочник. Киев: Наукова думка, 1990. - 224 с.

4. Биомеханические и материаловедческие свойства костной ткани [Электронный pecypc]. - URL: http://bone-surgery.ru/view/biomehanicheskie_i_materialovedcheskie_svojstva_kostnoj_tkani. (дата обращения: 12.09.2015).

5. Бранков Г. Основы биомеханики. - М.: Мир, 1981. - 256 с. 
6. Гаркавенко Ю.Е., Поздеев А.П. Ошибки и осложнения при удлинении нижней конечности у детей // Вестник гильдии протезистов-ортопедов. - 2002. - № 3. - С. 61-63.

7. Дашевский И.Н. Развитие модели разгрузки ортезов нижних конечностей // Сборник трудов VI Троицкой конференции «Медицинская физика и инновации в медицине» (ТКМФ-6). - Троицк, 2014. - C. 500-502.

8. Дашевский И.Н., Никитин С.Е. Экспериментальное изучение и биомеханическое моделирование функции разгрузки туторов нижних конечностей. - М., 2011. - 57 с.

9. Добелис М.А. Экспериментальное исследование механических свойств основных компонент костной ткани: автореф. дис. ... канд. техн. наук. - Рига, 1985. - 18 с.

10. Елизаров П.Е., Никитин С.Е., Лирцман В.М. Медицинская реабилитация больных с нестабильными переломами проксимального отдела плеча и их последствиями // Тезисы VII Городской науч.-практ. конф., посвящ. 40-летию городской больницы № 10, «Медицинская реабилитация пациентов с патологией опорно-двигательной и нервной систем». - М., 2006. - С. 194-195.

11. Зоря В.И., Васильев А.П., Корчебный Н.Н., Лужников И.Б. Лечение ложных суставов трубчатых костей у подростков и взрослых // Сб. тез. междунар. конгр. «Современные технологии в травматологии, ортопедии: ошибки и осложнения - профилактика и лечение», 05-07.10.2004, г. Москва. - М., 2004. - С. 36.

12. Карлов А.В., Шахов В.П. Системы внешней фиксации и регуляторные механизмы оптимальной биомеханики. - Томск: STT, 2001. - 477 с.

13. Каулен В.Д. Функциональное лечение диафизарных переломов голени укороченными гипсовыми повязками: автореф. дис. ... канд. мед. наук. - М., 1989.

14. Кашигина Е.А., Панфилов В.М., Никифорова Е.А. Функциональное лечение диафизарных переломов голени // Вестник Российского государственного медицинского университета. - 2003. - № 5 (31). C. 51-55.

15. Кейер А.Н., Рожков А.В. Руководство по протезированию и ортезированию. - СПб., 1999.

16. Ключевский В.В. Хирургия повреждений. - Ярославль, 1999. - С. 198-203.

17. Кораблева Н.Н., Викторов А.Е., Дубилей О.Ф., Глазков В.В., Голубев В.В., Скоробогатов А.М. Принципы ортезирования больных травматолого-ортопедического профиля ортезами фирмы «REHAND»// Тез. рос. науч.-практ. конф. «Ортезирование. Путь к совершенству». - М., 2002. C. 75-79.

18. Корж А.А., Меженина Е.П., Печерский А.Г., Рынденко В.Г. Справочник по травматологии и ортопедии. - Киев: Здоров'я, 1980. - 216 с.

19. Логвенков С.А., Штейн А.А. Управление биологическим ростом как задача механики // Российский журнал биомеханики. - 2006. - Т. 10, № 2. - С. 9-18.

20. Никитин С.Е. Ортезотерапия в системе лечения переломов костей конечностей и их последствий у взрослых: дис. ... д-ра. мед. наук. - М., $2011-261$ с.

21. Образцов И.Ф., Ханин М.А. Оптимальные биомеханические системы. - М.: Медицина, 1989. - 272 с.

22. Работнов Ю.Н. Механика деформируемого твердого тела. - М.: Наука. ГРФМЛ, 1979. - 744 с.

23. Руденко О.В., Сарвазян А.П. Волновая биомеханика скелетной мышцы // Акустический журнал. 2006. - Т. 52, № 1. - С. 720-732.

24. Сайт врача травмотолога-ортопеда С.Е. Никитина [Электронный ресурс]. - URL: http://senikitin.ru/orthosis.html. (дата обращения: 12.09.2015).

25. Тиманин Е.М., Еремин Е.В. Измерения модуля упругости бицепса человека средствами ультразвукового допплеровского эластографа // Сборник трудов V Троицкой конференции «Медицинская физика и инновации в медицине» (ТКМФ-5). - Троицк, 2012. - Т. 2. - С. 160-162.

26. ТУ 2244-022-03989419-02. Вспененный полиэтилен [Электронный ресурс]. - URL: http://www.avalot.ru/product/2.html. (дата обращения: 12.09.2015).

27. Федорова В.Н., Степанова Л.А. Краткий курс медицинской и биологической физики с элементами реабилитологии. Лекции и семинары: учеб. пособие. - М.: ФИЗМАТЛИТ, 2005. - 624 с.

28. Феодосьев В.И. Сопротивление материалов. - М.: Наука, 1986. - 512 с.

29. Физические параметры человека [Электронный ресурc]. - URL: http://www.t-z-n.ru/ presila/int_read13. html. (дата обращения: 12.09.2015).

30. Creylman V., Vander Sloten J., Peeraer L. Functional evaluation of ankle foot orthoses by finite element simulations // Proceedings of 17 IVO Congress, The Hague, Netherlands, November 13th and 14th, 2009. Programme. - Hague, 2009. - P. 94.

31. Creylman V., Vander Sloten J., Vermetten D., Peeraer L. Finite element analysis of ankle foot orthoses. Proceedings of international foot and ankle biomechanics community. - Bologna, Italy, 2008. - P. 137.

32. Fung Y.C. Biomechanics. Mechanical properties of living tissues. - New-York-Heidelberg-Berlin: SpringerVerlag, 1981. - 432 p. 
33. Gennisson J.-L., Catheline S., Chaffaï S., Fink M. Transient elastography in anisotropic medium: Application to the measurement of slow and fast shear wave speeds in muscles // J. Acoust. Soc. Amer. 2003. - Vol. 114, № 1. - P. 536-540.

34. Hsu J.D., Michael J.W., Fisk J.R. AAOS Atlas of orthoses and assistive devices. - Philadelphia: MosbyElsevier, 2008. - 652 p.

35. Jenkyn T.R., Ehman R.L., An K.N. Noninvasive muscle tension measurement using the novel technique of magnetic resonance elastography // J. Biomech. - 2003. - № 36. - P. 1917-1921.

36. Levinson S., Catheline S., Fink M. Anisotropic elasticity \& viscosity deduced from supersonic shear imaging in muscle // Annual Report Rochester Center for Biomedical Ultrasound. - Rochester, 2004. P. 17.

37. Muraru L., Creylman V., Pallari J. Validation of ankle foot orthosis finite element models by optical strain measurements [Электронный ресурс]. - URL: http://www.mobilab-khk.be/Publications/CMBBE2010_ LuizaMuraru_Poster.pdf. (дата обращения: 12.09.2015).

38. Poitout D.G., Dominique G. Biomechanics and biomaterials in orthopedics. - Berlin: Springer, 2004. - 654 p.

39. Wanamaker A.B. Biomechanical comparison of lower limb unloading between common modalities of ankle foot orthoses: Thesis. University of Denver. - Denver, 2013.

\section{BIOMECHANICS OF UNLOADING OF THE LOWER EXTREMITIES AT PROSTHETICS}

\section{I.N. Dashevskii, S.E. Nikitin (Moscow, Russia)}

It is studied the biomechanics of one type of modern lower extremity orthoses orthopedic devices enabling flexible adjustment to the individual patient during the whole treatment period that allows us to provide better fixation and unloading of the problem area, to reduce the time and improve the quality of treatment. Since overloading the damaged area is fraught with complications, and its underloading leads to weakening and atrophy of the musculoskeletal system and lengthening terms of rehabilitation, it is important the study of extremity unloading at orthotics, which is the main purpose of the study. Combined computational and experimental approach to the study of biomechanics of the system "footorthosis" is proposed for two types of orthoses. The effect of occurrence of lower extremity unloading at orthotics is demonstrated experimentally. We introduce a quantitative parameter of unloading - coefficient of unloading (CU), calculated as the ratio of the maximum load on an unorthosed extremity to the maximum load on the orthosed one. We describe direct experimental measurement in vivo of unloading coefficient using hardware and software complex "DiaSled" for two types of orthoses and with varying degrees of lateral compression (assessed qualitatively). For the coefficient of unloading, the values from 1 to about 2.3 were obtained, depending on the specific conditions. On the basis of comparative evaluations of stiffness of individual elements of "Leg-Orthosis" system, we proposed basic biomechanical model of extremity unloading at orthotics and derived the corresponding expression for CU. The model provides for $\mathrm{CU}$ an estimate of about 2.1-1.6 in the absence of lateral compression, and (depending on the contact conditions of the extremity with the orthosis) constancy or linear falling of the load on a extremity with increasing compression.

Key words: biomechanics of extremities, orthoses, tutors, lower extremities, experimental researches, unloading, DiaSled. 